Les canalopathies de la douleur chez l'homme

François Rugiero

La douleur

et ses différentes composantes

La douleur est un phénomène biologique complexe. Elle naît dans les terminaisons périphériques des neurones sensoriels (nocicepteurs) des ganglions des racines dorsales (DRG), est relayée dans la moelle épinière dorsale et transmise aux centres nerveux supérieurs (thalamus, cortex somato-sensoriel) le long du tractus spino-thalamique. Elle peut être grossièrement divisée en trois principales catégories d'un point de vue purement mécanistique, la nociception, la douleur inflammatoire aiguë et la douleur neuropathique. La nociception est une réaction physiologique qui permet à l'organisme d'éviter une situation qui pourrait être nocive ou dangereuse pour ses tissus. La nociception engage un circuit neuronal réflexe purement spinal, sans intervention des centres nerveux supérieurs. Un exemple de réflexe nociceptif est observé lorsqu'on pose sa main par mégarde sur un appareil très chaud et qu'on la retire instantanément sans penser afin de préserver l'intégrité de nos tissus dermiques. La nociception n'est pas à proprement parler une catégorie de douleur car la notion de douleur implique une composante consciente.

La définition officielle de la douleur, d'après l'association internationale pour l'étude de la douleur, est la suivante: «la douleur est une expérience sensorielle et émotionnelle déplaisante associée à une lésion tissulaire réelle ou potentielle ou décrite en termes d'une telle lésion» [1]. Cela signifie que le réflexe nociceptif est seulement la première étape de la réponse physiologique douloureuse. Lorsqu'on se brûle, on retire sa main de l'objet brûlant mais on assiste aussi au déclenchement d'une réaction inflammatoire en réponse à l'endommagement des tissus. C'est la deuxième principale composante de la douleur, que l'on appelle douleur inflammatoire aiguë. Elle est due à l'excitation des terminaisons nerveuses périphériques par des molécules pro-inflammatoires libérées par les tissus lésés et les cellules gliales et immunitaires environnantes. La douleur inflammatoire aiguë, comme le réflexe nociceptif, est une réaction physiologique nécessaire au maintien de l'intégrité de l'organisme. Elle résulte en une sensation douloureuse plus prononcée qu'habituellement (hyperalgésie). L'inflammation, bien que douloureuse, nous rappelle qu'il faut que nous soyons attentifs et que nous protégions nos tissus endommagés. Il est donc important sur un plan thérapeutique de ne pas trop empêcher la réaction inflammatoire aiguë de se dérouler normalement. Malheureusement il arrive que la douleur inflammatoire devienne chronique, par exemple en cas d'infection ou lors de syndromes auto-immuns, articulaires et gastro-entérologiques. II est dans ces cas nécessaire de la combattre.

Le troisième type de douleur, douleur neuropathique, implique une réaction qui n'est plus physiologique mais purement pathologique. Comme son nom l'indique, la douleur neuropathique est due non pas à une lésion des tissus qui entourent les terminaisons nerveuses sensorielles, mais à une lésion de ces mêmes nerfs. Ceci peut arriver lors de syndromes inflammatoires chroniques, d'une lésion mécanique des nerfs, par exemple une section ou un écrasement.
Wolfson Institute for Biomedical Research, University College London, Gower street, London WClE 6BT, Royaume-Uni.

f.rugiero@ucl.ac.uk

Près de $8 \%$ de la population mondiale est concernée par la douleur neuropathique, et pour $5 \%$ de la population la douleur est sévère [2]. Elle se manifeste notamment dans les neuropathies liées au diabète, lors de douleurs induites par les agents anticancéreux, de la fibromyalgie (ou syndrome polyalgique idiopathique diffus), de l'infection par le virus de l'herpès, le VIH (virus de l'immunodéficience humaine) dans le Sida, etc. La douleur neuropathique peut avoir une origine périphérique ou centrale ou bien souvent les deux. Elle crée une sensation de douleur lors de stimulations normalement inoffensives (allodynie).

\section{Les canalopathies}

La douleur étant un phénomène lié au système nerveux, il n'est pas étonnant que les principales cibles moléculaires pour la combattre se situent dans la catégorie des protéines transmembranaires qui régulent l'activité électrique des neurones sensoriels. Parmi celles-ci les canaux ioniques sont abondamment représentés [15]. Les canaux ioniques sont des protéines transmembranaires qui forment des pores dans la membrane cellulaire, permettant le passage des ions à travers celle-ci. Ils sont à la base de l'excitabilité neuronale et permettent le transfert d'informations d'un neurone à un autre par le biais de la genèse et de la conduction de potentiels d'action. On appelle canalopathies les conditions pathologiques qui sont associées au dysfonctionnement d'un canal ionique. De nombreuses canalopathies ont été mises en évidence au cours des dix dernières années $[3,4]$. Une même 


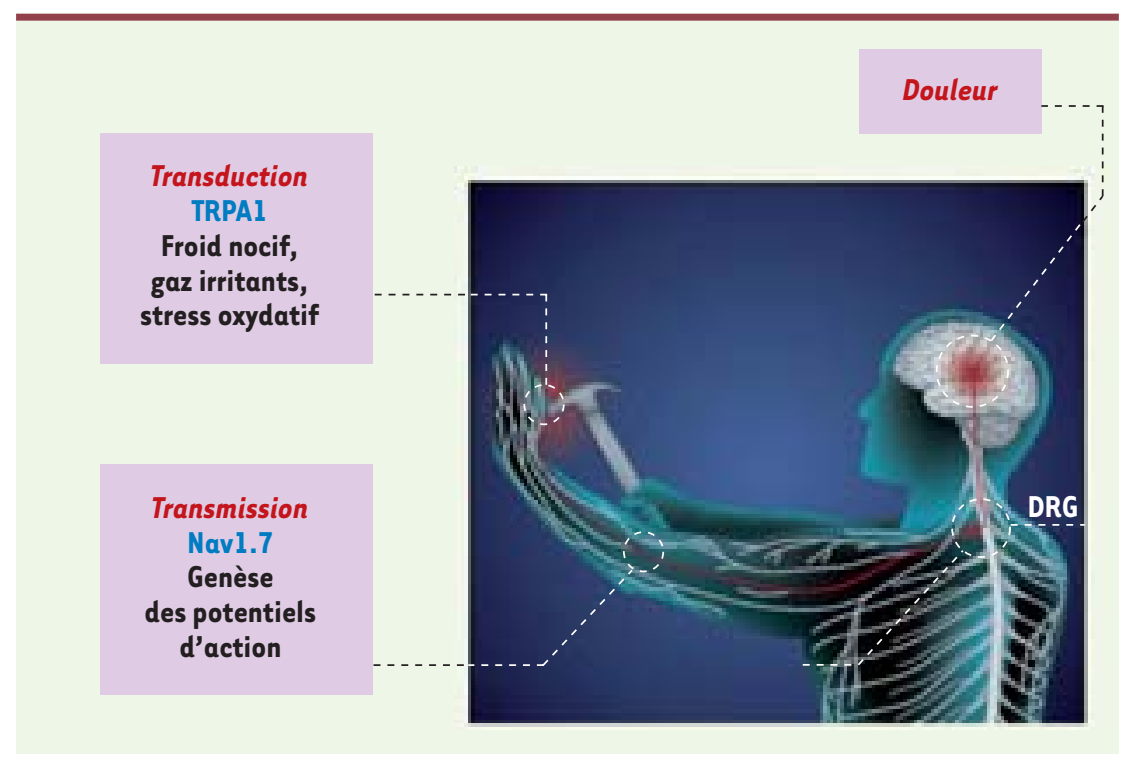

pathologie peut être causée par différentes mutations au sein d'un même gène codant pour un canal ionique. C'est le cas par exemple du canal chlore CFTR (cystic fibrosis transmembrane conductance regulator), dont de nombreuses mutations sont responsables de la fibrose kystique [5]. La majorité des canalopathies connues sont responsables de différentes formes d'épilepsies, de migraines, de problèmes cardiaques, musculaires et métaboliques [3, 4, 6]. Récemment, plusieurs mutations dans des canaux ioniques exprimés dans les neurones sensoriels ont été mises à jour et il a été démontré que ces mutations sont impliquées dans des symptômes de douleur chronique ou même dans l'absence complète de douleur chez l'homme.

\section{Les mutations impliquées dans} les syndromes douloureux chez l'homme Jusqu'à maintenant deux canaux ioniques seulement ont été répertoriés comme cibles de mutations entraînant des syndromes douloureux chez l'homme. II s'agit du canal sodium Navl.7 (voltagegated sodium channel subunit alpha) ${ }^{1}$, exprimé dans les neurones sensoriels et

${ }^{1}$ Navl.7 est aussi nommée: sodium channel protein type 9 subunit alpha, SCN9A. les neurones sympathiques, et du canal cationique TRPAl (transient receptor potential cation channel subfamily $A$ member 1), fortement exprimé dans les neurones sensoriels mais présent également dans d'autres neurones et types cellulaires.

\section{Mutations dans le canal sodium Navl.7}

Les premières mutations identifiées en 2004 dans le canal Navl.7 sont des mutations faux sens dominantes responsables d'une condition douloureuse appelée érythromélalgie primaire familiale $(P E)$ : c'est une maladie causée par la chaleur et caractérisée par des épisodes de douleur insupportable accompagnée de rougeur et gonflement des pieds et parfois des mains [7]. Dans la majorité des cas ces mutations ont pour effet de rendre l'activation du canal sodium Navl.7 plus facile (en hyperpolarisant son potentiel d'activation) et de ralentir sa désactivation. Sachant que Navl.7 est impliqué dans l'amplification des potentiels de récepteur dans les DRG, il en résulte une hyperexcitabilité des neurones de DRG possédant le canal muté. Un deuxième syndrome douloureux lié à des mutations dans le gène de Navl.7 est le syndrome de douleur paroxystique extrême (PEPD, paroxysmal extreme pain disorder). Cette condition se caractérise par des douleurs

Figure 1. Canaux ioniques dont les mutations sont responsables de syndromes douloureux chez l'homme. II s'agit du canal cationique TRPAl (transient receptor potential cation channel subfamily A member 1) et du canal sodium Navl.7 (voltage-gated sodium channel subunit alpha). Ces canaux sont exprimés dans les neurones sensoriels (nocicepteurs) des ganglions des racines dorsales (DRG) : Navl.7 est impliqué dans l'amplification des potentiels de récepteur dans les DRG (transmission), et TRPAl est exprimé dans une souspopulation de nocicepteurs et sert de récepteur au froid extrême. La douleur est relayée dans la moelle épinière dorsale et transmise aux centres nerveux supérieurs (thalamus, cortex somato-sensoriel).

périrectales, péri-oculaires et périmandibulaires qui peuvent être déclenchées par une stimulation de la région péri-anale, par exemple lors de la défécation, ou par le froid, la prise d'aliments ou une situation émotionnelle [8]. Les mutations impliquées sont également des mutations gain de fonction, dominantes, ciblant cette fois l'inactivation du canal. Cette dernière est entravée, ce qui entraîne une certaine persistance du courant lors de son activation par dépolarisation de la membrane plasmique [8]. Bien que les effets ne soient pas complets, la carbamazépine peut représenter une thérapie du syndrome PEPD [9], contrairement à l'érythromélalgie primaire familiale pour laquelle aucune thérapie n'existe pour l'instant. L'existence de ces deux syndromes douloureux prouve le rôle critique que joue le canal Navl.7 dans la genèse de la sensation douleureuse. Si l'on suit cette logique, on peut s'attendre à ce qu'une mutation dont l'effet est d'abroger l'activation de Navl.7 supprime la sensation douloureuse. C'est exactement ce qui a été démontré en 2006 quand trois mutations faux sens distinctes dans Navl.7 ont été découvertes chez des individus insensibles à la douleur [10]. Ces trois mutations rendent le canal complètement inopérant, et les individus atteints complètement insensibles à la douleur bien que leurs nerfs soient intacts. Ces personnes finissent souvent par mourir à un âge 
relativement jeune car l'absence de douleur leur enlève aussi la notion de peur. Ainsi peuvent-elles sauter du haut d'un toit et se casser un membre ou bien se couper un bras sans rien sentir et mourir des suites de ces blessures. Les patients atteints d'insensibilité congénitale à la douleur prouvent ainsi l'importance physiologique critique de la douleur pour la survie des individus. Malgré les promesses de la thérapie génique, aucune solution thérapeutique n'existe encore.

\section{Mutations dans le canal TRPAl}

Dernièrement nous avons identifié un nouveau canal ionique à l'origine d'un syndrome congénital douloureux. Il s'agit du canal TRPAl, de la famille des canaux TRP (transient receptor potential) qui sont largement exprimés dans les divers systèmes sensoriels [11] et eux-mêmes impliqués dans de nombreuses canalopathies [12]. TRPAl est exprimé dans une sous-population de nocicepteurs et sert de récepteur au froid extrême douloureux, à de nombreux composés actifs naturels que l'on trouve dans la moutarde, le poivre, l'ail, l'oignon, la cannelle, etc., ainsi qu'à divers irritants (gaz lacrymogènes, etc.) et au stress oxydatif. Nous avons identifié une mutation dans la séquence de TRPAl (N855S) qui, chez l'homme, est responsable d'un syndrome douloureux épisodique, FEPS (familial episodic pain syndrome) [13], qui se manifeste par des attaques de douleur au niveau thoracique qui durent en moyenne une heure et demie, s'accompagnent de difficultés respiratoires, tachycardie, transpiration, pâleur, cyanose péri-buccale et dureté de l'abdomen et se déclenchent en réponse à une combinaison de paramètres, fatigue, jeûne et froid. La douleur engendrée par la mutation N855S s'explique par l'ouverture du canal TRPAl au potentiel de repos des neurones sensoriels, ce qui est normalement rare [13]. L'ouverture de TRPAl au repos entraîne une accumulation de charges positives à l'intérieur des neurones, ce qui les dépolarise et les rend hyperexcitables. Les individus atteints de ce syndrome sont rares, comme les patients qui ont une insensibilité à la douleur, et ils vivent dans des régions reculées où la consanguinité dans les familles est encore importante.

Une différence importante entre FEPS et $P \varepsilon / P E P D$ se situe dans l'expression des canaux. L'expression de Navl.7 est relativement restreinte (DRG et neurones sympathiques) et explique aisément que des mutations touchant ce canal altèrent la sensation douloureuse, un phénomène généré dans les DRG. TRPAl au contraire est exprimé dans un grand nombre de tissus différents et la localisation précise des symptômes de la mutation N855S dans les neurones des DRG est pour l'instant inexpliquée. II est possible que l'effet localisé de la mutation N855S soit dû à l'interaction de TRPAl avec d'autres canaux spécifiques aux DRG. Une observation importante réside dans le fait que le canal TRPAl muté peut être bloqué par un inhibiteur spécifique de TRPAl, HC-030031, ce qui laisse penser qu'une solution thérapeutique à cette condition pourrait être mise au point rapidement.

La liste des canalopathies liées à la douleur est encore très courte [14] mais le champ d'étude de ces conditions s'est considérablement élargi au cours des dix dernières années grâce à l'avancement des techniques de séquençage de I'ADN, ce qui laisse penser que d'autres mutations dans des canaux ioniques situés dans les circuits neuronaux de la douleur seront probablement mises à jour dans le futur. $\diamond$ Human pain channelopathies

\section{CONFLIT D'INTÉRÊTS}

L'auteur déclare n'avoir aucun conflit d'intérêts concernant les données publiées dans cet article.

\section{RÉFÉRENCES}

1. International association for the study of pain. Subcommittee on taxonomy. The need of a taxonomy. Pain $1979 ; 6: 247-52$.

2. Torrance N, Smith BH, Bennett MI, Lee AJ. The epidemiology of chronic pain of predominantly neuropathic origin. Results from a general population survey. J Pain 2006 ; 7 : 281-9.

3. Kass RS. The channelopathies: novel insights into molecular and genetic mechanisms of human disease. J Clin Invest 2005; 115: 1986-9.

4. Kullmann DM. Neurological channelopathies. Annu Rev Neurosci 2010 ; 33 : 151-72.

5. Gadsby DC, Vergani P, Csanády L. The ABC protein turned chloride channel whose failure causes cystic fibrosis. Nature $2006 ; 440: 477-83$.

6. Cregg R, Momin A, Rugiero F, et al. Pain channelopathies. J Physiol 2010; 588 : 1897-904.

7. Yang $Y$, Wang $Y$, Li S, et al. Mutations in SCN9A, encoding a sodium channel alpha subunit, in patients with primary erythermalgia. J Med Genet 2004 ; 41 : 171-4.

8. Fertleman CR, Baker MD, Parker KA, et al. SCN9A mutations in paroxysmal extreme pain disorder: allelic variants underlie distinct channel defects and phenotypes. Neuron $2006 ; 52: 767-74$

9. Fertleman CR, Ferrie CD, Aicardi J, et al. Paroxysmal extreme pain disorder (previously familial rectal pain syndrome). Neurology 2007 ; 69 : 586-95.

10. Cox JJ, Reimann F, Nicholas AK, et al. An SCN9A channelopathy causes congenital inability to experience pain. Nature $2006 ; 444: 894-8$.

11. Damann N, Voets T, Nilius B. TRPs in our senses. Curr Biol 2008 ; 18 : R880-9.

12. Nilius B, Owsianik G. Transient receptor potential channelopathies. Pflug Arch $2010 ; 460: 437-50$.

13. Kremeyer B, Lopera F, Cox JJ, et al. A gain-of-function mutation in TRPAl causes familial episodic pain syndrome. Neuron $2010 ; 66: 671-80$.

14. Waxman SG. Channelopathic pain: a growing but still small list of model disorders. Neuron $2010 ; 66: 622-4$.

15. Weiss N, De Waard M. Les canaux calciques dépendants du voltage au cœur de la douleur. Med Sci (Paris) 2006 ; 22 : 396-404.

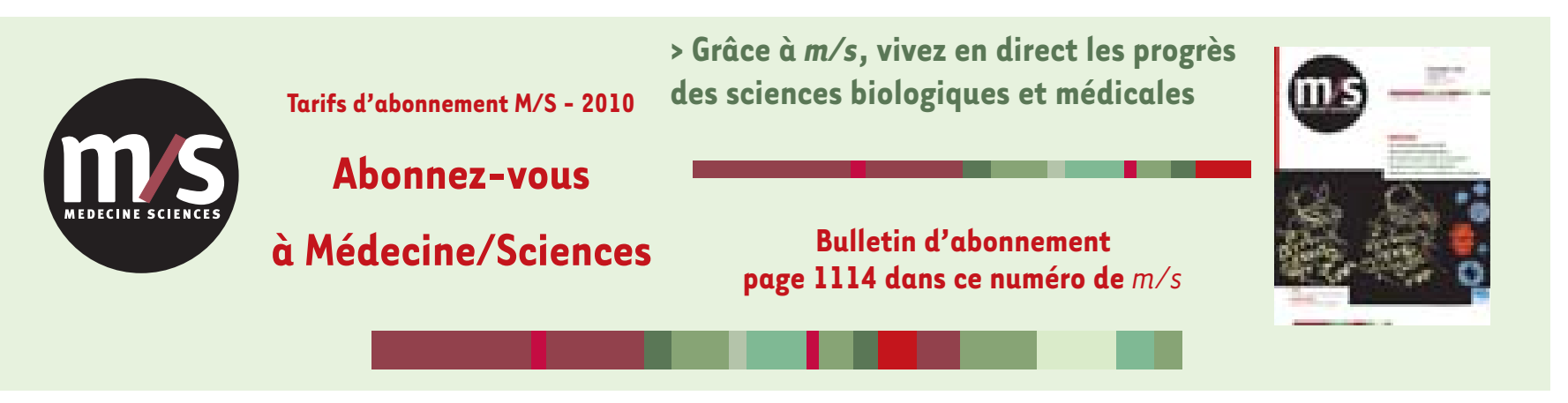

